\title{
Minor Body Science with the Nancy Grace Roman Space Telescope
}
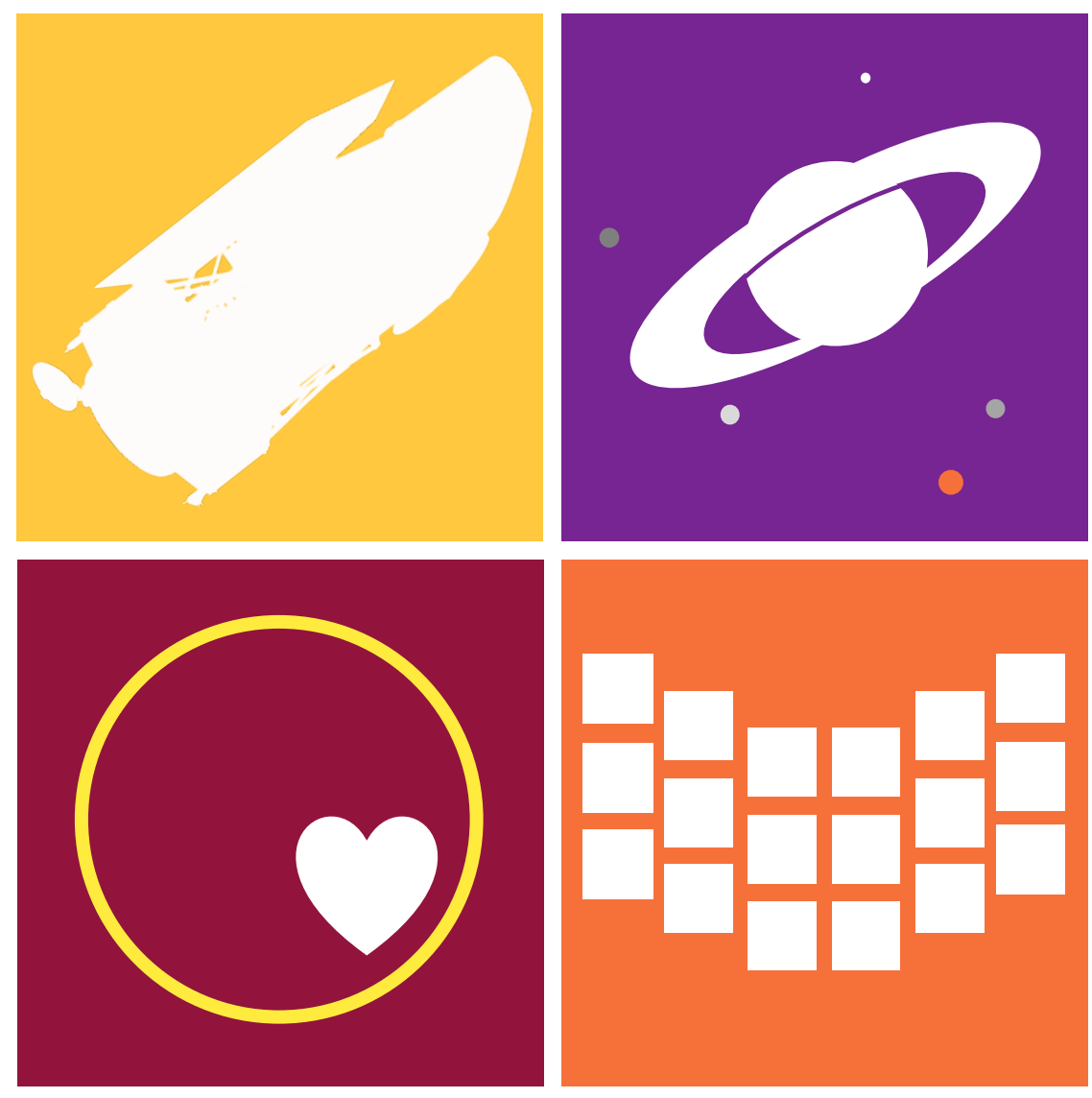

Bryan J. Holler (STScI)*, Stefanie N. Milam (NASA/GSFC), James M. Bauer (U. Maryland), Jeffrey W. Kruk (NASA/GSFC), Charles Alcock (Harvard/CfA), Michele T. Bannister (U. of Canterbury), Gordon L. Bjoraker (NASA/GSFC), Dennis Bodewits (Auburn), Amanda S. Bosh (MIT), Marc W. Buie (SwRI), Tony L. Farnham (UMD), Nader Haghighipour (Hawaii/IfA), Paul S. Hardersen (Unaffiliated), Alan W. Harris (MoreData! Inc.), Christopher M. Hirata (Ohio State), Henry H. Hsieh (PSI, Academica Sinica), Michael S. P. Kelley (U. Maryland),

Matthew M. Knight (USNA/U. Maryland), Emily A. Kramer (Caltech/JPL), Andrea Longobardo (INAF/IAPS), Conor A. Nixon (NASA/GSFC), Ernesto Palomba (INAF/IAPS), Silvia Protopapa (SwRI), Lynnae C. Quick (Smithsonian), Darin Ragozzine (BYU), Jason D.

Rhodes (Caltech/JPL), Andy S. Rivkin (JHU/APL), Gal Sarid (SETI, Science Systems and Applications, Inc.), Amanda A. Sickafoose (SAAO, MIT), Cristina A. Thomas (NAU), David E. Trilling (NAU), Robert A. West (Caltech/JPL)

*Contact information for primary author: Bryan J. Holler (STScI), bholler@stsci.edu, (667) 218-6404

Thematic areas: Ground- and space-based telescopes, Primitive bodies 


\section{Solar system science with space telescopes}

There is a long history of solar system observations with space telescope facilities, from the Infrared Astronomical Satellite (IRAS) in the 1980s to present-day facilities such as the Hubble Space Telescope. Some of these facilities include a prominent solar system component as part of the original mission plan (e.g., WISE, JWST), some included this component late in mission design or even after primary operations begin (e.g., HST), and others never intended to support solar system observations until the proper opportunity arose (e.g., Kepler, Chandra). Nevertheless, the solar system community finds a way to make use of these facilities for groundbreaking science. The time allocation for solar system observations is typically not that high, yet they make a disproportionately large impact. According to [1], 2.3\% of HST orbits between 2014 and 2017 were devoted to solar system observations, while $15.4 \%$ of the press releases in that time span were related to these projects (Figure 1). Solar system science has proven to be a powerful tool for generating interest in space telescopes among the general public and will continue to do so for next-generation facilities.

HST Orbit Breakdown (Cycles 22-24)

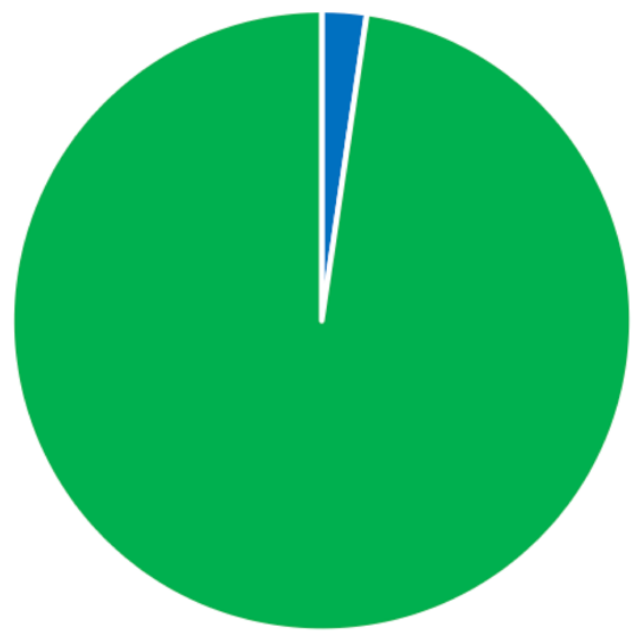

- Solar System Other
HST Press Release Breakdown (2015-2017)

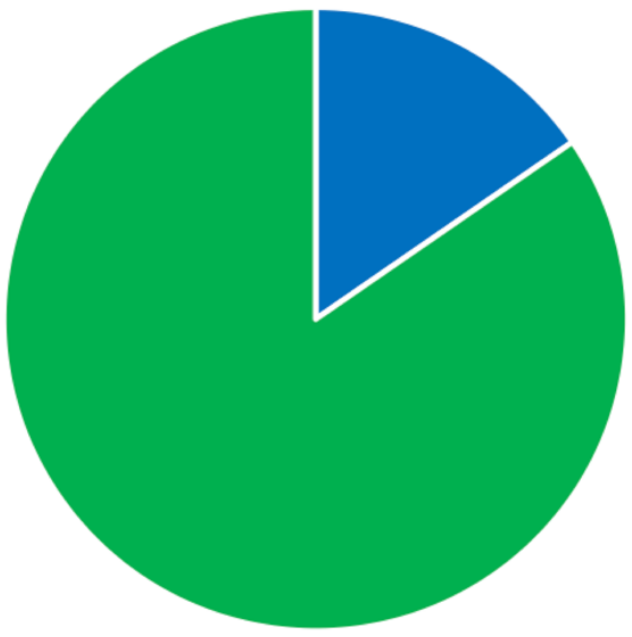

- Solar System " Other

Figure 1: (left) Pie chart of orbit breakdown by science category for Cycles 22-24 (Oct. 1, 2014-Sept. 30, 2017). Solar system observations (blue) accounted for $\sim 2.3 \%$ of the total orbits in this time span. (right) Pie chart of the press release breakdown by science category for the period covering 2015-2017 (Cycles 22-24). Solar system science accounts for a disproportionately large fraction of the number of press releases (and therefore public outreach and awareness) compared to the orbits granted for solar system observing programs: $\sim 15.4 \%$ vs. $\sim 2.3 \%$. (From [1].)

These next-generation facilities will have some combination of larger apertures, larger fields of view, and more sensitive instrumentation than their predecessors. The prospects for solar system science with future facilities are substantial, particularly in the area of space-based surveys for the detection of minor bodies. In the past decade alone there have been numerous advances in the study of minor bodies, some with space-based facilities and others with groundbased facilities, including the first detections of objects in previously uncharacterized populations. 
Such discoveries include the first Earth Trojan asteroid [2], the first interstellar object [3], and the first object orbiting the Sun beyond 100 au [4]. An especially interesting aspect of Earth's Trojans is their potential for future manned spaceflight missions due to their proximity to Earth. As discussed in [1], the Nancy Grace Roman Space Telescope, formerly the Wide Field InfraRed Survey Telescope (WFIRST) and referred to hereafter as "Roman," has a field of regard (range of solar elongation angles available to the telescope) that reaches angles as small as $54^{\circ}$, and thus would be capable of carrying out a survey for additional Earth Trojans. In general, a future space-based survey mission, ideally with a large field of view and deep-imaging capabilities, would provide the means to discover more minor bodies in new and intriguing populations.

Targeted solar system surveys are not necessarily required to make advances in solar system science. Surveys focused on astrophysical targets such as exoplanets, supernovae, and galaxies can provide the coverage and depth needed to identify and characterize foreground minor bodies. Part of the Roman mission is to execute Core Community Surveys that will include a high (galactic) latitude survey, a survey for extragalactic transients, and a time series survey of the galactic bulge. These surveys would contribute to serendipitous detections of minor bodies over a large range of heliocentric distances with higher efficiency and greater depth than many previous targeted ground-based surveys.

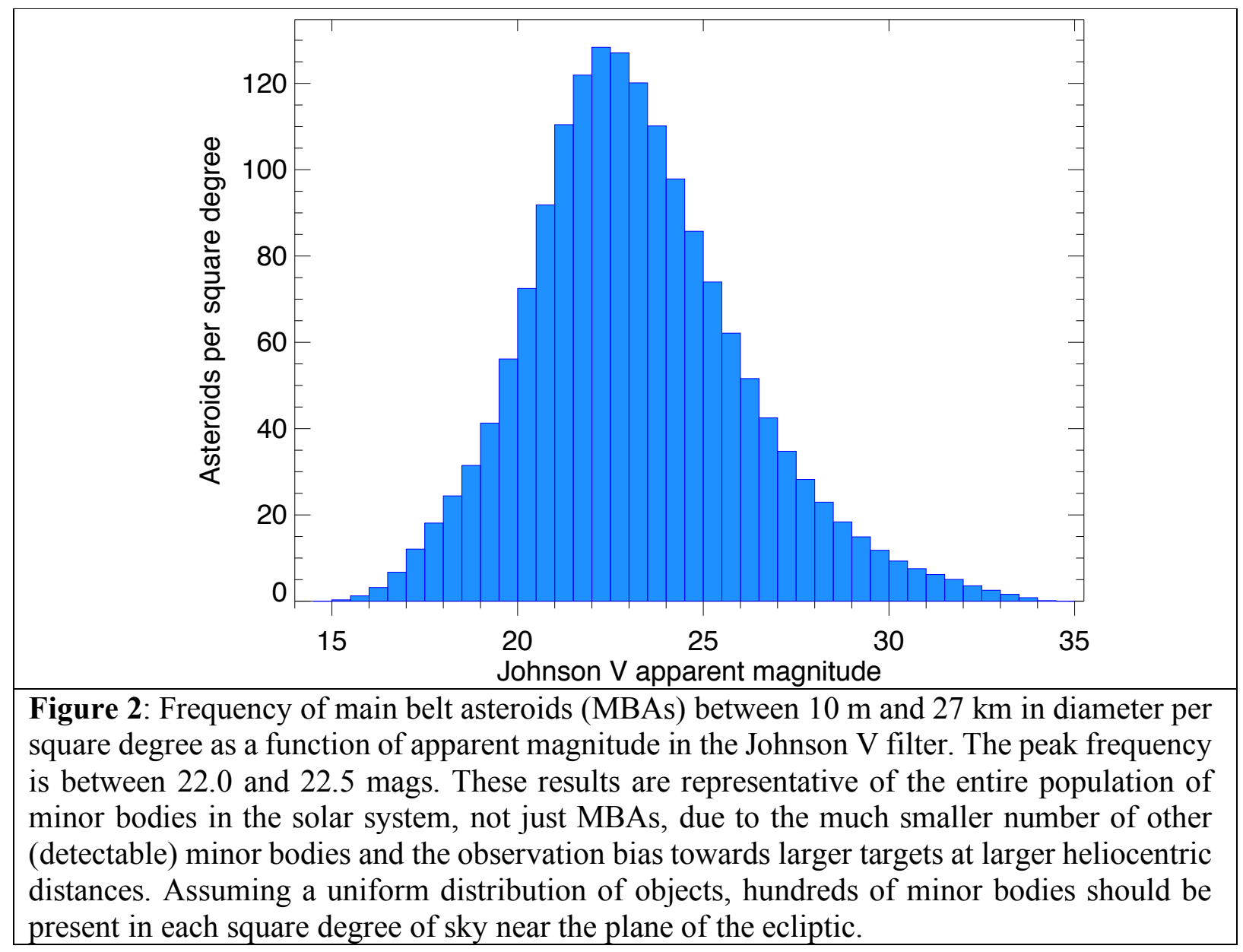

As shown in Figure 2, hundreds of minor bodies, including NEAs, main belt asteroids (MBAs), and Kuiper Belt Objects (KBOs), are present in each square degree near the ecliptic. A mission with a sufficiently large field of view and deep imaging capabilities, such as Roman, 
would be best able to take advantage of this high target density [5]. The prospects for serendipitous detection of minor bodies is highly dependent on mission and survey parameters and would therefore benefit significantly from contributing to their definition. For the majority of this white paper, we will discuss the prospects for two hypothetical targeted surveys using Roman, which has deep imaging capabilities (i.e., sensitive instrumentation and wide-band filters) and a wide field of view $\left(0.28 \mathrm{deg}^{2}\right)$. These two surveys would deliver high-impact minor body science by moving the inventory of irregular satellites (captured minor bodies) around the giant planets closer to completion and expanding on the number of known Inner Oort Cloud objects beyond $100 \mathrm{au}$. Each of these populations contributes a different piece to the puzzle of the early dynamical evolution of our solar system.

\section{Irregular satellite surveys}

The irregular satellites of the giant planets are a diverse collection of minor bodies thought to have been captured early in the history of the solar system. While almost all irregular satellites are thought to be the result of capture, the source populations are still debated. They may have originated from extant or primordial minor body populations, but their exact origins remain uncertain due largely to the small number currently known $(\sim 125)$. Further frustrating efforts at determining irregular satellite origins are their wide range of orbital parameters and the lack of constraints on physical characteristics and surface compositions. At this point in time, in situ spacecraft observations actually provide our best views of irregular satellites, but to-date only two have been visited: Saturn's moon Phoebe [6] and Neptune's moon Triton [7]. Both objects appear to have their origins in the Kuiper Belt, but it is also clear from these limited observations that not all irregular satellites fit this paradigm, implying multiple source populations.

In an effort to better understand their origins, the irregular satellite populations of Jupiter and Saturn were categorized into "families" based on their orbital properties (inclination, eccentricity, semi-major axis, direction of orbital motion). Common origins, either from the same source population or through collisional processes, were identified for irregular satellite families through this process [8-12]. Probing the Hill spheres of the giant planets (the volume of space where that planet's gravity dominates over the Sun's and satellites can have stable orbits) for additional small, faint irregular satellites would move the inventories of these populations closer to completion and help to further inform origin theories.

Roman is expected to be able to reach a limiting magnitude of $\mathrm{V} \sim 28$ in a reasonable exposure time ( $\sim 1000$ seconds), so irregular satellites down to approximately $0.3,1.0,4.5$, and $11.4 \mathrm{~km}$ in diameter could be detected around Jupiter, Saturn, Uranus, and Neptune, respectively. We used these diameter limits in combination with an estimated size distribution supported by observations $[13,14]$ to predict the number of irregular satellites detectable with Roman. With no preference for irregular satellites on prograde or retrograde orbits, and assuming only half of the unknown irregular satellites larger than the diameters estimated above are discovered, we would expect to identify approximately 1000, 200, 100, and 5 new irregular satellites with Roman around Jupiter, Saturn, Uranus, and Neptune, respectively. Compare these numbers to the 71, 58, 9, and 7 currently known irregular satellites around each of the giant planets. This steep increase is because current surveys are unable to detect the more numerous collisionally produced irregular satellites, which are smaller and fainter.

The search efficiency for new irregular satellites would benefit significantly from Roman's large field of view $\left(0.28 \mathrm{deg}^{2}\right)$. The search regions of interest around the giant planets correspond to the angular extent of each planet's Hill sphere; the solid angle of the giant planets' 
Hill spheres as seen from Earth are 4.7, 3.0, 1.5, and $1.5 \mathrm{deg}^{2}$, respectively [15]. However, dynamical stability studies by [16] find that satellites are not truly stable over the entire volume of the Hill sphere; the maximum semi-major axis for an irregular satellite on a retrograde orbit is only $\sim 67 \%$ of the Hill radius. This fraction is even smaller for an object in a prograde orbit. Table 1 presents the size of this "stability region", as well as the semi-major axis of the furthest currently known irregular satellite around each giant planet. Even given this stability constraint, a very large fraction of the stable volume of each giant planet's Hill sphere remains to be searched; this region also covers larger angular distances from the primary, so scattered light from the giant planet is likely to be less of an issue. This is not surprising given the small field of view of the ground-based telescopes typically used to carry out these surveys and the difficulty in obtaining the observing time necessary to image the entirety of the Hill spheres to an appropriate depth for detection of the smallest irregular satellites.

Jupiter's Hill sphere has the largest angular extent on the sky and the field of view of Roman $\left(0.28 \mathrm{deg}^{2}\right)$, for example, could cover the entire area in only 17 pointings, compared to the nearly 2000 pointings for an imager with a field of view 3 arcminutes on a side. What is currently a prohibitively long survey could be undertaken in only a few hours with Roman, enabling plenty of follow-up opportunities to further improve the orbital solutions of any currently known or newly discovered irregular satellites.

Table 1. Giant planet Hill spheres

\begin{tabular}{|c|c|c|c|c|}
\hline Planet & $\begin{array}{c}\text { Hill radius } \\
\left(\mathbf{1 0}^{\mathbf{7}} \mathbf{~ k m}\right)\end{array}$ & $\begin{array}{c}\text { Stability region } \\
\left.\mathbf{( 1 0}^{\mathbf{7}} \mathbf{~ k m}\right)\end{array}$ & $\begin{array}{c}\text { Semi-major axis of } \\
\text { furthest known } \\
\text { satellite } \mathbf{( 1 0} \mathbf{~ k m )}\end{array}$ & $\begin{array}{c}\text { \% vol. of Hill } \\
\text { sphere known } \\
\text { to be occupied }\end{array}$ \\
\hline Jupiter & 5.32 & 3.56 & 2.86 & 52 \\
\hline Saturn & 6.53 & 4.38 & 2.45 & 18 \\
\hline Uranus & 7.00 & 4.69 & 2.09 & 9 \\
\hline Neptune & 11.6 & 7.77 & 4.93 & 26 \\
\hline
\end{tabular}

A survey to detect Inner Oort Cloud objects

Minor bodies at the edge of the solar system are time capsules that preserve the history of the formation of the solar system, and the distribution of their orbits may provide clues to the existence of a potential, distant giant planet [17]. These distant minor bodies, especially those in the "Inner Oort Cloud" ( $\mathrm{r} \gtrsim 100 \mathrm{au}$ ) [18] and the Oort Cloud proper ( $\mathrm{r} \gtrsim 1000 \mathrm{au}$ ), provide valuable compositional information on the original solar nebula but, typically, observers must wait until they make the extraordinarily long journey into the inner solar system in order to detect and study them. The size of the Inner Oort Cloud population is very poorly constrained and must be constrained to better understand the formation and dynamical evolution of the early solar system.

At the time of writing, only two objects have been confirmed at heliocentric distances $>100$ au: $2018 \mathrm{VG}_{18}$, at $\sim 125$ au [4] and the object nicknamed "FarFarOut," reported via press release in February 2019 at $\sim 140$ au. A handful of other objects have been identified with orbits that result in them spending significant periods of time beyond $100 \mathrm{au}$, but none of these detections were made when the objects were beyond 100 au [19-21]. This is due to the extremely large distances involved, making their angular motion on the sky exceedingly small, as well as the physical characteristics of the objects themselves: Small sizes and dark surfaces combine to produce very low reflected fluxes (Figure 3). Thus, space-based observations using instruments with high sensitivities and large quantities of observing time are necessary to 
detect small bodies in the Inner Oort Cloud.

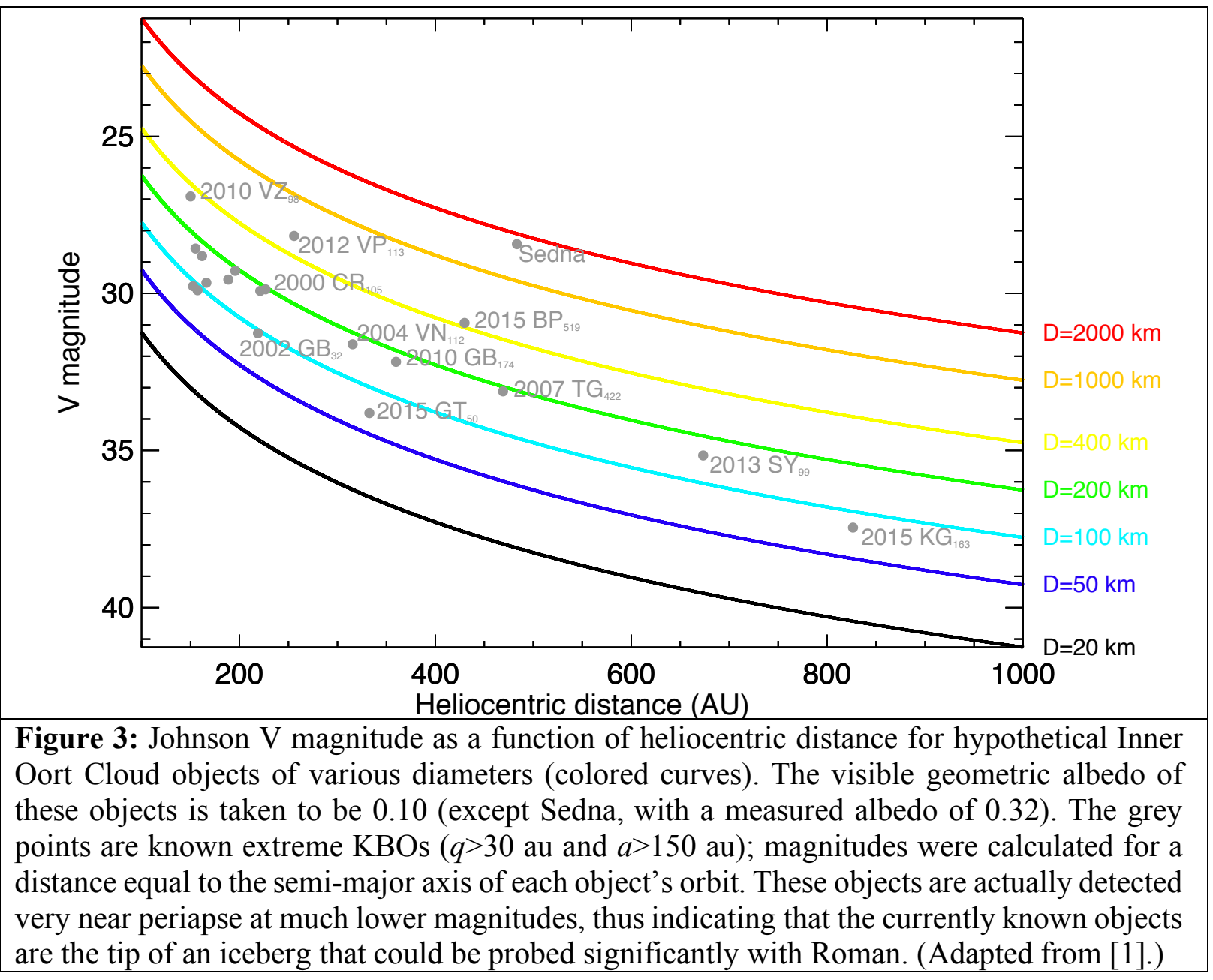

Very long exposures of 1000s of seconds or more are required to detect distant, faint Inner Oort Cloud objects, but there is a practical limit for exposure length set by cosmic rays. According to [22], the flux of cosmic rays at the Sun-Earth L2 point is $\sim 3.3 \mathrm{~cm}^{-2} \mathrm{~s}^{-1}$ averaged over a full solar cycle, assuming a shielded focal plane. For the Roman Wide Field Imager (WFI) parameters for pixel area $\left(100 \mu \mathrm{m}^{2}\right)$ and physical detector area $\left(\sim 300 \mathrm{~cm}^{2}\right)$, and assuming only one pixel is affected by each cosmic ray strike, $\sim 0.33 \%$ of pixels would be affected in a 1000 -second exposure. However, it is more realistic to assume that the pixel hit by the cosmic ray and each adjacent pixel would be affected, meaning that $\sim 1.65 \%$ would be affected in 1000 seconds.

Given this information, we now outline a hypothetical survey with the dual goals of identifying Inner Oort Cloud objects and surveying faint astrophysical sources that may not be detected as part of the Core Community Surveys, such as very high-redshift galaxies. The survey strategy is to obtain ten 1000 -second exposures at one pointing, then return at a later time $(<1$ day) and obtain ten 1000-second exposures of a second field offset from the first. Overlapping the two fields by $\sim 25 \%$ provides deeper imaging over a reasonable amount of the first field while still increasing the total survey area. This process would be continued over days to years in order to build up a large, deep survey region. Compared to the isotropic distribution of comets in the Oort Cloud [23], the shape of the Inner Oort Cloud is possibly more disk-like [18], centered on 
the ecliptic, with a larger spread in ecliptic latitude as heliocentric distance increases. This results in a higher density of objects over a smaller area of sky, increasing the chances of Inner Oort Cloud objects present in any particular pointing. An ideal region for the survey would be within a few tens of degrees of the ecliptic plane, outside regions heavily populated with low-redshift galaxies, and off the galactic plane in order to avoid source confusion.

An object at the lower end of the range (100 au) will move $\sim 4$ pixels (at $\sim 0.1$ " per pixel) over 10,000 seconds and $\sim 32$ pixels over the course of one day, whereas objects at 600 au will only move $\sim 2$ pixels over the course of one day. Re-imaging quadrants of earlier fields over the span of a few days would therefore allow for detection of objects over a large range of heliocentric distances, including 1000s of KBOs. Use of the large field of view of Roman would increase the survey area at a faster rate compared to many currently operating ground- or spacebased telescopes and would open a whole new frontier in the study of our solar system.

\section{Serendipitous detection of active minor bodies}

Comets are remainders from the time of the formation of the giant planets and serve as a probe of primitive composition. To estimate the number of comets available to Roman, we repeated the James Webb Space Telescope (JWST) non-sidereal rate study from [24] using Roman's solar elongation constraints. In brief, they considered all known comets with perihelion dates between January 2010 and January 2015, independent of their discovery circumstances: 221 short-period and 172 long-period comets. We find that Roman can observe a greater fraction of comets than JWST due to the wider field of regard $\left(54^{\circ}-126^{\circ}\right.$ versus $\left.85^{\circ}-135^{\circ}\right)$, despite the same assumed rate limit of $\mathbf{3 0} \mathbf{~ m a s} / \mathrm{s}$. In order to establish lower bounds, we neglect activity and assume bare cometary nuclei albedos are $\sim 5 \%$, similar to some other outer solar system populations [25]. This means that Roman, with magnitude limits approaching V=26 or more, will be capable of detecting objects down to $\sim 1 \mathrm{~km}$ beyond Jupiter's orbit serendipitously in the Core Community Surveys and General Observer (GO) observations, or as part of targeted minor body surveys. Roman's ability to detect comets of all types at larger heliocentric distances offers the unique capability to study bare nuclei without the typical obscuration of coma, and to follow the development of activity as the comet approaches the Sun. The total population of Jupiter Family Comets $(\mathrm{D} \geq 1.5 \mathrm{~km})$ is estimated to be 2000 [26], of which 584 have been numbered (https://physics.ucf.edu/ yfernandez/cometlist.html).

Active asteroids [27] are a classification of minor bodies that possess asteroid-like dynamical properties but exhibit mass loss. They include main-belt comets (MBCs) [28], which orbit in the main asteroid belt and whose dust emission is due to the sublimation of volatile ice, and disrupted asteroids, whose dust emission is due to physically disruptive processes such as impacts or rotational destabilization rather than sublimation. MBCs present an opportunity to study the nature, extent, and abundance of ice in inner solar system bodies and have implications for understanding the formation of our solar system and delivery of water to the early Earth. Disrupted asteroids present opportunities to study disruptive processes on small bodies in real time for comparison to theoretical models of those processes and also for inferring properties of their interiors. Discovery of more such objects [29] is crucial for advancing our understanding of their abundance and distribution in the inner solar system and the ranges of various physical properties associated with these objects (e.g., sizes, activity levels, active lifetimes, rotation periods, colors, etc.). Serendipitous detection of active asteroids will be conducted using data obtained as part of the already planned Roman high-latitude and microlensing surveys for follow-up with JWST and ground-based observatories. 
Two interstellar objects have been discovered since 2017: 1I/ Oumuamua [3] and 2I/Borisov. Both objects are only a few hundred meters in diameter and therefore are difficult to detect unless they pass through the inner solar system; it is expected that thousands are currently within the orbit of Neptune at any given time [30]. However, this and similar estimates may be off by orders of magnitude based on small number statistics, and only the detection of additional objects will help improve the accuracy of future estimates. Evaluating the compositions of interstellar objects (volatile content, dust grain size, $\mathrm{D} / \mathrm{H}$ ratio, etc.) presents unique opportunities to directly constrain the compositions of other proto-planetary nebulae for comparison to our own solar system's formation environment. The potential compositional diversity of this population was demonstrated through the different behaviors of 1I and 2I. Both exhibited non-gravitational accelerations [31-33] but only 2I/Borisov produced an observable coma, with direct measurements of volatile CO [34-36]. Due to their extremely high velocities, interstellar objects spend considerably less time in the inner solar system than bound objects with comparable perihelion distances. Earlier detection of interstellar objects at larger distances will expand opportunities for follow-up with other facilities, both ground- and space-based. The WFI on Roman, with its large field of view and sensitivity, would be crucial to this effort as a complementary facility to the Vera C. Rubin Observatory (formerly the Large Synoptic Survey Telescope, LSST), which can only observe fields in the southern sky. Due to interstellar object origins in other stellar systems, their distribution in the sky is expected to be isotropic, so a survey in only one hemisphere or only along the ecliptic misses at least half the sky. The high (galactic) latitude Core Community Survey and the Inner Oort Cloud survey (proposed above) would provide excellent coverage and depth for serendipitous detection of transient interstellar objects.

\section{References}

[1] Holler, B.J., et al. 2018. JATIS 4, 034003. [2] Connors, M., et al. 2011. Nature 475, 481-483. [3] Meech, K.J., et al. 2017. Nature 552, 378-381. [4] Sheppard, S.S., et al. 2018. MPEC 2018Y14. [5] Gould, A. 2014. JKAS 47, 279-291. [6] Johnson, T.V., Lunine, J.I. 2005. Nature 435, 6971. [7] Agnor, C.B., Hamilton, D.P. 2006. Nature 441, 192-194. [8] Gladman, B., et al. 2001. Nature 412, 163-166. [9] Nesvorný, D., et al. 2003. AJ 126, 398-429. [10] Sheppard, S.S., Jewitt, D.C. 2003. Nature 423, 261-263. [11] Jewitt, D., Haghighipour, N. 2007. ARAA 45, 261-295. [12] Holt, T.R., et al. 2018. ApJ 859, 97. [13] Nicholson, P.D., et al. 2008. The Solar System Beyond Neptune, pp. 411-424. [14] Bottke, W.F., et al. 2010. AJ 139, 994-1014. [15] Sheppard, S.S. 2006. IAUS 229, pp. 319-334. [16] Hamilton, D.P., Krivov, A.V. 1997. Icarus 128, 241-249. [17] Batygin, K., Brown, M.E. 2016. AJ 151, 22. [18] Hills, J.G. 1981. AJ 86, 1730-1740. [19] Brown, M.E., et al. 2004. ApJ 617, 645-649. [20] Trujillo, C.A., Sheppard, S.S. 2014. Nature 507, 471474. [21] Trujillo, C., et al. 2018. DPS 50, \#311.09. [22] Robberto, M. 2010. JWST-STScI-001928, SM-12. [23] Dones, L., et al. 2004. Comets II, pp. 153-174. [24] Kelley, M.S.P., et al. 2016. PASP 128, 018009. [25] Grav, T., et al. 2012. ApJ 744, 197. [26] Bauer, J.M., et al. 2017. AJ 154, 53. [27] Jewitt, D., et al. 2015. Asteroids IV, pp. 221-241. [28] Hsieh, H.H., Jewitt, D. 2006. Science 312, 561-563. [29] Hsieh, H.H., et al. 2015. Icarus 248, 289-312. [30] Jewitt, D., et al. 2017. ApJL 850, L36. [31] Micheli, M., et al. 2018. Nature 559, 223-226. [32] Hui, M.-T., et al. 2020. arXiv:2003.14064. [33] Jewitt, D., et al. 2020. ApJL 888, L23. [34] Ye, Q., et al. 2020. AJ 159, 77. [35] Bodewits, D., et al. 2020. Nat. Ast. doi:10.1038/s41550-020-1095-2. [36] Cordiner, M.A., et al. 2020. Nat. Ast. doi:10.1038/s41550-020-1087-2. 